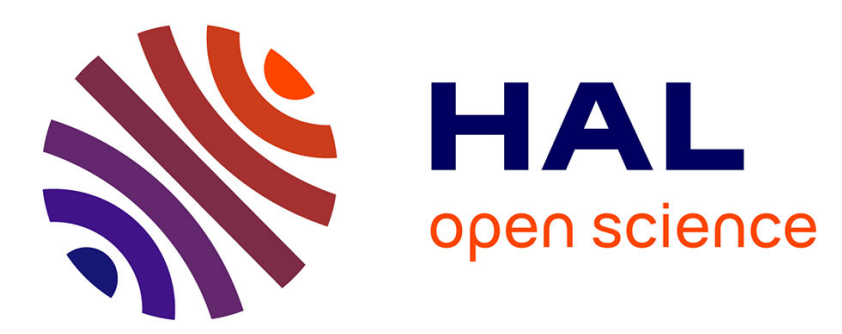

\title{
The Atlantic side of the Iberian Peninsula: a hot-spot of novel African honey bee maternal diversity
}

Maria Pinto, Irene Muñoz, Julio Chávez-Galarza, Pilar Rúa

\section{To cite this version:}

Maria Pinto, Irene Muñoz, Julio Chávez-Galarza, Pilar Rúa. The Atlantic side of the Iberian Peninsula: a hot-spot of novel African honey bee maternal diversity. Apidologie, 2012, 43 (6), pp.663-673. 10.1007/s13592-012-0141-1 . hal-01003663

\section{HAL Id: hal-01003663 https://hal.science/hal-01003663}

Submitted on 1 Jan 2012

HAL is a multi-disciplinary open access archive for the deposit and dissemination of scientific research documents, whether they are published or not. The documents may come from teaching and research institutions in France or abroad, or from public or private research centers.
L'archive ouverte pluridisciplinaire HAL, est destinée au dépôt et à la diffusion de documents scientifiques de niveau recherche, publiés ou non, émanant des établissements d'enseignement et de recherche français ou étrangers, des laboratoires publics ou privés. 


\title{
The Atlantic side of the Iberian Peninsula: a hot-spot of novel African honey bee maternal diversity
}

\author{
Maria Alice Pinto ${ }^{1}$, Irene Muñoz ${ }^{2}$, Julio Chávez-GalarZa ${ }^{1}$, Pilar De la Rúa ${ }^{2}$ \\ ${ }^{1}$ Mountain Research Centre (CIMO), Polytechnic Institute of Bragança, Campus de Sta. Apolónia, \\ Apartado 1172, 5301-855 Bragança, Portugal \\ ${ }^{2}$ Área de Biologia Animal, Dpto. de Zoología y Antropología Física, Facultad de Veterinaria, \\ Universidad de Murcia, Campus de Espinardo, 30100 Murcia, Spain
}

Received 10 November 2011 - Revised 14 April 2012 - Accepted 2 May 2012

\begin{abstract}
The Iberian honey bee has been one of the most surveyed subspecies for genetic diversity. Yet, previous studies have missed an important component of Iberian honey bee variation harbored by populations inhabiting the Atlantic side of the Iberian Peninsula. Herein, we provide a fuller picture of the Iberian honey bee maternal diversity by revealing 16 novel haplotypes detected in honey bees from Portugal. Of the 16 haplotypes, all of African ancestry, 15 belong to the Atlantic sub-lineage $\mathrm{A}_{\text {III }}$ while only one fits the most common sub-lineage $A_{I}$. This level of new variation is remarkable as it represents a $59 \%$ increase in the widerange African lineage and a $188 \%$ in sub-lineage $\mathrm{A}_{\mathrm{III}}$. Our findings further highlight the complexity of the Iberian honey bee diversity patterns and reinforce the importance of this southernmost European territory as a reservoir of Apis mellifera genetic diversity, a resource increasingly important in a rapidly changing and demanding world.
\end{abstract}

\section{Iberian honey bee / genetic diversity / mtDNA / DraI test / Portugal}

\section{INTRODUCTION}

Understanding patterns and underlying processes of diversity of the western honey bee (Apis mellifera L.) has been a major goal of numerous genetic studies, which in a population declining scenario is becoming increasingly important because it can provide a stronger scientific basis for management and conservation decisions. Among the 29 recognized honey bee subspecies (Engel 1999; Sheppard and Meixner 2003), the

Electronic supplementary material The online version of this article (doi:10.1007/s13592-012-0141-1) contains supplementary material, which is available to authorized users.

Corresponding author: M.A. Pinto, apinto@ipb.pt

Manuscript editor: Marina Meixner
Iberian honey bee has been one of the most intensively surveyed for diversity patterns. Indeed, studies of Iberian honey bees have been performed since the 1970s (Ruttner et al. 1978) using morphology (Cornuet and Fresnaye 1989; Arias et al. 2006; Miguel et al. 2010), allozymes (Smith and Glenn 1995; Arias et al. 2006), mitochondrial DNA (Smith et al. 1991; Garnery et al. 1995, 1998a; Franck et al. 1998; De la Rúa et al. 2001, 2004, 2005; Arias et al. 2006; Miguel et al. 2007; Cánovas et al. 2008), and microsatellites (Franck et al. 1998; Garnery et al. 1998b; De la Rúa et al. 2002, 2003; Miguel et al. 2007; Cánovas et al. 2011). Differential and complex patterns of diversity have emerged from these surveys, which have yet to be fully resolved.

The maternally inherited mitochondrial DNA (mtDNA) has been the marker of choice for 
assessing Iberian honey bee variation, particularly the PCR-RFLP of the intergenic tRNA ${ }^{\text {leu }}$ cox2 region, also known as the DraI test (Garnery et al. 1993). Over 2,500 colonies, mostly sampled in the eastern half of the Iberian Peninsula, have been screened with the DraI test (Garnery et al. 1993, 1995, 1998a; Miguel et al. 2007; Cánovas et al. 2008). The data generated by this massive sampling confirmed early findings of coexistence of African (A) and western European (M) lineages, forming a south-north cline (Smith et al. 1991), and revealed unparalleled levels of haplotype diversity and complexity (Franck et al. 1998; Cánovas et al. 2008). Accordingly, it has been suggested that, as for other animal and plant species, the Iberian Peninsula served as a glacial refuge (Hewitt 1999, 2001; Arias et al. 2006; Gómez and Lunt 2007), and as a place of secondary contact between European and African evolutionary lineages (Smith et al. 1991; Garnery et al. 1995; Cánovas et al. 2008), and therefore it has been a stage for historical processes possibly involving recurring phenomena of local adaptation, contraction, fragmentation, expansion, and admixture.

In contrast with the populations inhabiting the eastern side of the Iberian Peninsula, Portuguese honey bees have been largely undersampled. Yet, the few samples collected in Portugal (Garnery et al. 1998a; Arias et al. 2006; Miguel et al. 2007) suggest that the Atlantic side of Iberia may harbor an important component of the Iberian honey bee maternal diversity. Therefore, a fuller understanding of diversity patterns of the Iberian honey bee requires further surveys of Ibero-Atlantic populations. As part of an ongoing genetic study of the honey bees occupying the Portuguese territory, we have detected 16 novel haplotypes with the $\operatorname{DraI}$ test. This finding is non-trivial as this small corner of southern Europe is contributing with $59 \%$ of new variation to the widerange African lineage. Herein, the novel haplotypes are fully described by the RFLP approach and by sequence data. Our findings suggest that the Atlantic side of the Iberian Peninsula harbors important genetic resources, especially in face of the escalating threats to the honey bee diversity.

\section{MATERIALS AND METHODS}

\subsection{Samples and RFLP analysis}

As part of an ongoing study of the Portuguese honey bee populations, over 950 stationary colonies were sampled, between 2008 and 2010, covering every district of continental Portugal and the archipelagos of Azores and Madeira (sample sizes and locations are provided in Online resource 1). Honey bee workers were collected from the inner part of the hives, placed in absolute ethanol, and then stored at $-20^{\circ} \mathrm{C}$ until molecular analysis. The maternal ancestry of the 950 workers, each representing a single colony and a single apiary, was assessed using the DraI test (Garnery et al. 1993), which consists on PCR amplification of the $\mathrm{RNNA}^{\text {leu }}$-cox 2 intergenic region, using the primers E2 and $\mathrm{H} 2$ (see PCR details in Garnery et al. 1993), followed by digestion with the restriction enzyme DraI (see digestion and gel electrophoresis details in Cánovas et al. 2008). Of the 950 individuals scored using the complete set of restriction maps and restriction fragment sizes reported to date (De la Rúa et al. 1998, 2005; Franck et al. 2001; Collet et al. 2006), 43 exhibited a total of 16 unreported PCR-RFLP patterns. Location of the 43 colonies, from which the individuals were sampled, is shown in Figure 1. In this study, the 16 novel PCR-RFLP patterns were fully characterized by sequencing.

\subsection{Sequencing and sequence analysis}

The tRNA ${ }^{\text {leu }}$-cox 2 intergenic region analyzed herein contains a non-coding sequence which size depends on the forms of the $\mathrm{P}$ element and the number of repeats of the $\mathrm{Q}$ element. In the African lineage, the $\mathrm{P}$ element can display two different forms: $P_{0}$ (sub-lineage $A_{I}$ and $A_{I I}$ ) and $P_{1}$ (sublineage $A_{\text {III }}$ ). The $P_{0}$ differs from $P_{1}$ by a $15-b p$ deletion. The $\mathrm{Q}$ element can be repeated in tandem one to four times. A further distinction between sub- 


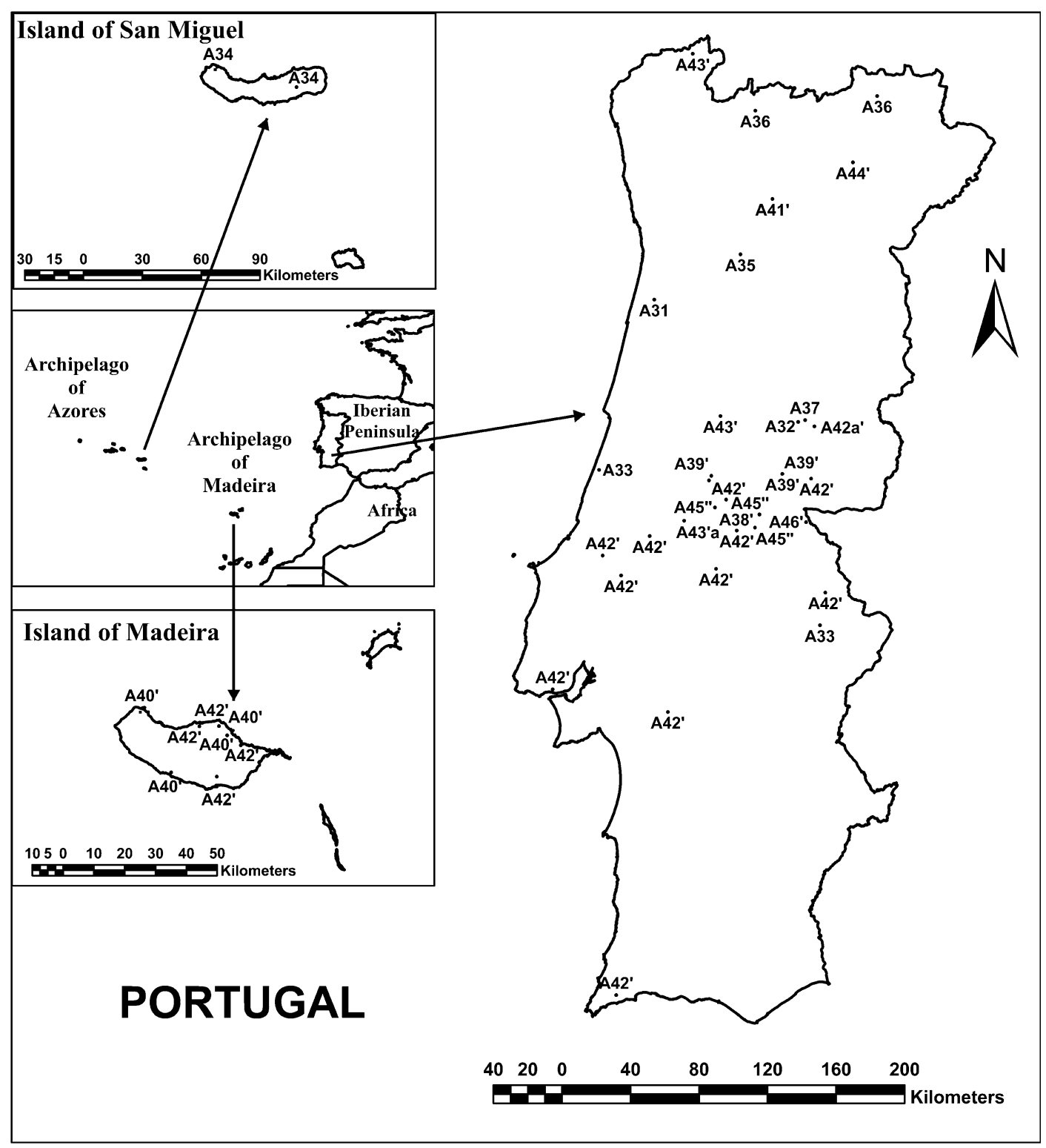

Figure 1. Location of the 43 colonies harboring the 16 novel PCR-RFLP haplotypes identified with the DraI test.

lineages $A_{I}$ and $A_{I I}$ is provided by the number of DraI recognition sites in the region spanning tRNA ${ }^{\text {leu }}$ and $5^{\prime}$ end of the first Q element, with two and one sites, respectively (Garnery et al. 1993; De la Rúa et al. 1998; Franck et al. 1998).

The 16 novel PCR-RFLP patterns were further examined by sequencing the tRNA ${ }^{\text {leu }}$-cox 2 intergenic region for 20 individuals. In addition, three individuals exhibiting the previously reported haplotypes A3 (sub-lineage $\mathrm{A}_{\mathrm{I}}$ ), A14, and A16 (sub-lineage $\mathrm{A}_{\text {III }}$ ) were also sequenced for comparison. Following PCR amplification, PCR products were purified either with isopropanol and ammonium acetate or using a column-based purification kit 
(Zymo Research ${ }^{\circledR}$ ) and sent to Secugen S. L. (Madrid, Spain) or Macrogen (Seoul, Korea) for direct sequencing in both directions with primers E2 and H2. The sequences were checked for base calling using SeqMan ${ }^{\circledR}$ version 7.0.0 and then deposited in GenBank (http://www.ncbi.nlm.nih.gov).

The 23 sequences, plus the published sequences of haplotypes A29a (FJ890930.1; Szalanski and Magnus 2010) and A30 (EF033654.1; Collet et al. 2006), belonging to sub-lineage $\mathrm{A}_{\text {III }}$, were aligned using MEGA version 5.03 (Tamura et al. 2011). Proximity among the haplotypes was established using the median-joining network algorithm (Bandelt et al. 1999) as implemented in the program Network version 4.6.0.0 (Fluxus Engineering, Clare, UK; http://www.fluxus-engineering.com), with epsilon set to zero and downweighting the four most variable sites (weight $=0$ for the most variable site; weight $=5$ for the remaining three most variable sites). Indels (e.g., $\mathrm{P}_{1}$ element, third and fourth $\mathrm{Q}$ element, 15deletion of $\mathrm{A}^{4} 0^{\prime}$ ) were considered as a single mutational step, being therefore coded as a 1-bp gap. Variable characters within the first (Q1), second (Q2), and third Q (Q3) elements were included in the analysis.

\section{RESULTS}

The DraI test performed on colonies surveyed across Portugal (continent and archipelagos of Azores and Madeira) identified 16 novel PCR-RFLP patterns (haplotypes) carried by 43 colonies (Figure 1). The restriction maps and length of restriction fragments (Figure 2) suggest that they all fit within the African evolutionary lineage (A). Following the nomenclature established earlier (Garnery et al. 1998a) and recently reviewed for lineage $M$ (Rortais et al. 2011), the 16 haplotypes were numbered sequentially from A31 to A46 (accession numbers JQ746684-JQ746701). Haplotypes showing the same RFLP pattern but bearing three or four Q elements were further distinguished by addition of the symbols ' and ", respectively, after the haplotype number (Garnery et al. 1998a; Rortais et al. 2011).

Figure 2 shows that the 16 patterns are dramatically distinct from those previously reported for the African lineage. The number of restriction fragments varied between three (A31, A41', A45") and five (A32, A35, A37, $\mathrm{A} 40^{\prime}$ ), with most haplotypes exhibiting a fourfragment pattern (A33, A34, A36, A38', A39', A42', A43', A44', A46'). The shortest band (28 bp) was displayed by four haplotypes (A35, A36, A37, A39') whereas the longest (1,064 bp) was unique to A45".

Location of the 43 colonies, and corresponding distribution of the 16 haplotypes depicted in Figure 1, shows that haplotype A42' was the most widespread and common (16 colonies) followed by $\mathrm{A} 40^{\prime}$ (four colonies). Haplotypes A39', A43', and A45" were carried by three colonies whereas A33, A34, and A36 by two colonies. The remaining eight haplotypes were singletons (A31, A32, A35, A37, A38', A41', A44', and A46'). While most haplotypes were detected in the center of continental Portugal, A34 and A40' were private to the islands of San Miguel (Azores) and Madeira, respectively. A42' was the only haplotype detected in both mainland and island (Madeira) populations.

Sequence data (Figure 3) confirmed the novelty of the 16 haplotypes of African ancestry and allowed identification of two additional variants of haplotypes A42' and A43', which were distinguished by a lowercase letter (A42'a, A43'a), as suggested by Rortais et al. (2011). Among the 16 haplotypes, 15 contained the $\mathrm{P}_{1}$ element whereas only one (A46') exhibited the $\mathrm{P}_{0}$ element. The length of the sequenced region ranged from $803 \mathrm{bp}$ to $1,204 \mathrm{bp}$, depending on the number of $\mathrm{Q}$ elements and presence of indels. Fifteen haplotypes contained either two (A31, A32, A33, A34, A35, A36, A37) or three Q elements (A38', A39', A40', A41', A42', A43', A44', A46'). Only one haplotype (A45") displayed a sequence with four $\mathrm{Q}$ elements (Figure 2). As typical for this intergenic region, numerous indels of variable sizes were detected within the $\mathrm{P}$ and $\mathrm{Q}$ elements (Figure 3). In addition to the 15-bp deletion characteristic of the $\mathrm{P}_{1}$ element (marked as " $d 1$ " in Figures 2 and 3), large indels were displayed by A31, A34, $\mathrm{A} 40$ ', and $\mathrm{A}^{4} 1^{\prime}$. The largest fragment $(33 \mathrm{bp}$, marked as "c" in Figure 3) was inserted in the 


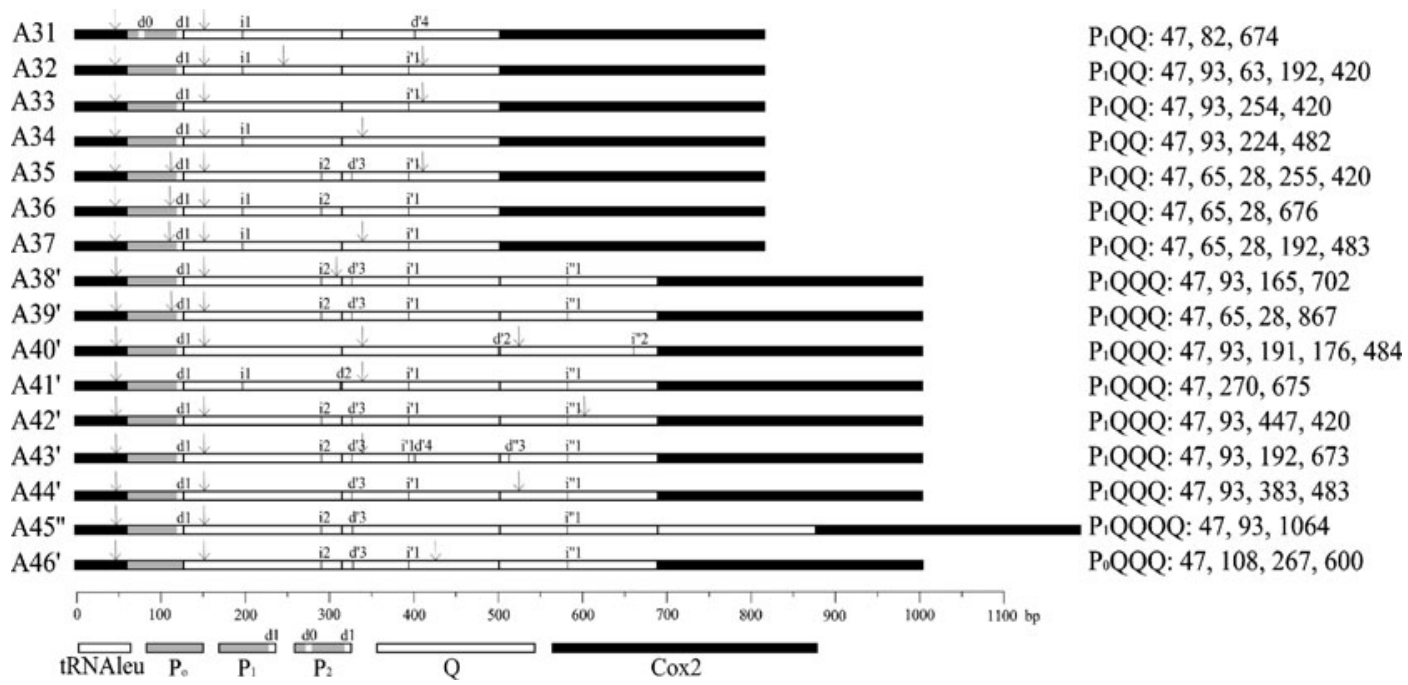

Figure 2. Restriction maps (left) and length of restriction fragments (right), deduced from DraI restriction patterns and sequences of the tRNA ${ }^{\text {leu }}$-cox2 intergenic region, of the 16 novel haplotypes found in Portugal. The DraI recognition site is denoted with an arrow. Deletions and insertions are numbered preceded by the letters $d$ and $i$, respectively. Deletion marked as $d 1$ is specific of the $\mathrm{P}_{1}$ element which defines sub-lineage $\mathrm{A}_{\mathrm{III}}$, as in Franck et al. (2001).

Q1 element of haplotype A34. This 33-bp insertion produced the longest $\mathrm{Q}$ element (227 bp) ever reported. The 15-bp deletion motif of A40' (marked as "l" in Figure 3) and A41' (marked as "h" in Figure 3) was a perfect match of $d l$, the only difference being the position. Indeed, while $d 1$ was located at the $3^{\prime}$ end of the $\mathrm{P}$ element, deletions of $\mathrm{A} 41^{\prime}$ and A40' were at the $3^{\prime}$ end of Q1 and Q2, respectively. In addition to the variation originated from large indels, there were 10 short indels (1-2 bp) and 17 single-base substitutions, of which nine resulted in a gain/loss of the DraI recognition site. The most striking mutation, displayed by seven haplotypes, is the " $\mathrm{AG}$ " inserted at the 3' end of Q1 element (marked as "f" in Figure 3). Interestingly, except for the Q3 of haplotype A41', no other Q element carried this mutation.

A median-joining network (Figure 4) based on 36 variable sites (18 coded indels and 18 substitutions) illustrates the relationships among the novel and previously described haplotypes of sub-lineages $\mathrm{A}_{\mathrm{I}}(\mathrm{A} 3)$ and $\mathrm{A}_{\mathrm{III}}$ (A14, A16, A29a, A30). Two distinct clusters, separated by the number of $\mathrm{Q}$ elements, are represented in the network. The more poorly resolved right-hand side cluster connects the haplotypes with two Q elements. One unresolved connection and four hypothetical haplotypes (unsampled or extinct) link this group to $\mathrm{A} 35$, the closest haplotype of the left-hand side cluster. Haplotypes A36 and A37 are the closest in the group with only one mutational step (a transition in A36 that led to loss of DraI restriction site) separating them. The haplotype pairs A30/A37 and A32/A33 are separated by one hypothetical haplotype and two mutational steps (one is a transversion that led to a gain/ loss of DraI restriction site). The previously described A14 and A34, which are derived from the same hypothetical haplotype, are the most distant in the group. A minimum of eight mutations, including the duplication of the $\mathrm{Q}$ element, and two hypothetical nodes separate the closest haplotypes of both clusters (A35 and A43'a).

The better resolved left-hand side cluster connects the haplotypes with three and four Q elements. The 14 haplotypes are separated from each other by one to eight mutational steps and three hypothetical nodes. The previously de- 


\begin{tabular}{|c|c|c|c|c|c|c|c|c|c|c|c|c|c|c|c|c|c|c|c|c|c|c|c|c|c|c|c|c|c|c|c|c|c|c|c|}
\hline & & & 78 & 79 & 80 & 81 & 82 & 83 & 84 & 85 & 86 & 102 & 111 & 112 & 115 & 116 & 117 & 118 & 119 & 120 & 121 & 122 & 123 & 1241 & 1251 & 1261 & 127 & 1281 & 129 & 158 & 197 & 198 & 199 & 200 & 201 \\
\hline & $\mathbf{a}$ & & & & & & & & & & & 1 & 2 & 3 & $d 1$ & & & & & & & & & & & & & & & 4 & $\mathrm{c}$ & & & & \\
\hline A3 & $\mathrm{A}$ & $\mathrm{T}$ & A & A & A & A & $\mathrm{T}$ & A & A & A & A & $\mathrm{T}$ & $\mathrm{T}$ & $\mathrm{A}$ & $\mathrm{A}$ & $\mathrm{T}$ & $\mathrm{T}$ & $\mathrm{T}$ & A & A & $\mathrm{T}$ & $\mathrm{T}$ & $\mathrm{T}$ & A & $\mathrm{T}$ & $\mathrm{T}$ & A & A & A & A & . & & & & - \\
\hline A14 & & & A & A & A & A & $\mathrm{T}$ & $\mathrm{A}$ & $\mathrm{A}$ & $\mathrm{A}$ & & C & $\mathrm{T}$ & A & & & & & & & & & & & & & & & - & A & - & - & - & - & - \\
\hline A16 & $F$ & & A & A & A & A & $\mathrm{T}$ & A & A & A & A & $\mathrm{T}$ & $\mathrm{T}$ & A & - & & - & & & & . & & - & - & . & - & - & & - & A & - & - & - & - & - \\
\hline A29a & A & $\mathrm{T}$ & A & A & A & A & $\mathrm{T}$ & A & A & A & A & $\mathrm{T}$ & $\mathrm{T}$ & A & - & - & - & - & & & - & - & - & 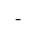 & - & - & - & & - & A & - & - & - & - & - \\
\hline A30 & A & $\mathrm{T}$ & A & A & A & A & $\mathrm{T}$ & A & A & A & A & $\mathrm{T}$ & $\mathrm{T}$ & A & - & . & . & - & & . & - & & - & & & - & - & & - & G & - & - & - & - & - \\
\hline A31 & . & - & - & - & - & - & - & - & - & - & - & $\mathrm{T}$ & $\mathrm{T}$ & A & - & - & - & - & - & & - & & - & - & . & - & . & . & & A & - & - & - & - & - \\
\hline A3 & A & $\mathrm{T}$ & A & A & A & A & $\mathrm{T}$ & A & A & A & A & $\mathrm{T}$ & $\mathrm{T}$ & A & - & - & - & - & - & - & - & - & - & - & - & - & - & - & - & A & - & - & - & - & - \\
\hline A33 & A & $\mathrm{T}$ & A & A & A & A & $\mathrm{T}$ & A & A & A & A & $\mathrm{T}$ & $\mathrm{T}$ & A & - & - & - & - & - & - & - & - & - & - & - & - & - & - & - & A & - & - & - & - & - \\
\hline A34 & A & $\mathrm{T}$ & A & A & A & A & $\mathrm{T}$ & A & A & A & A & C & A & A & - & - & - & - & - & - & - & - & - & - & - & - & - & - & - & A & $\mathrm{T}$ & $\mathrm{T}$ & $\mathrm{T}$ & A & $\mathrm{T}$ \\
\hline A35 & A & $\mathrm{T}$ & A & A & A & A & $\mathrm{T}$ & A & A & A & A & $\mathrm{T}$ & $\mathrm{T}$ & $\mathrm{T}$ & - & - & - & - & - & - & - & - & - & - & & - & - & & - & A & - & - & - & - & - \\
\hline A36 & A & $\mathrm{T}$ & A & A & A & A & $\mathrm{T}$ & A & A & A & A & $\mathrm{T}$ & $\mathrm{T}$ & $\mathrm{T}$ & - & - & - & - & - & - & - & 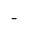 & - & & - & - & - & & - & A & - & - & - & - & - \\
\hline A37 & A & $\mathrm{T}$ & A & A & A & A & $\mathrm{T}$ & A & A & A & A & $\mathrm{T}$ & $\mathrm{T}$ & $\mathrm{T}$ & - & - & - & - & - & - & - & - & - & - & - & - & - & - & - & A & - & - & - & - & - \\
\hline $\mathbf{A 3 8}^{\prime}$ & A & $\mathrm{T}$ & A & A & A & A & $\mathrm{T}$ & A & A & A & A & $\mathrm{T}$ & $\mathrm{T}$ & A & - & - & - & - & - & - & - & - & - & - & - & - & - & - & - & A & - & - & - & - & - \\
\hline A39' & A & $\mathrm{T}$ & A & A & A & A & $\mathrm{T}$ & A & A & A & A & $\mathrm{T}$ & $\mathrm{T}$ & $\mathrm{T}$ & - & - & - & - & - & - & - & - & - & - & $\ldots$ & - & - & - & - & A & - & - & - & - & - \\
\hline A40 & A & $\mathrm{T}$ & A & A & A & A & $\mathrm{T}$ & A & A & A & A & $\mathrm{T}$ & $\mathrm{T}$ & A & - & - & - & - & - & - & - & - & - & - & - & - & - & - & - & A & - & - & - & - & - \\
\hline A41 & A & $\mathrm{T}$ & A & A & A & A & $\mathrm{T}$ & A & A & A & A & $\mathrm{T}$ & $\mathrm{T}$ & A & - & - & - & - & - & - & - & - & - & - & - & - & - & - & - & G & - & - & - & - & - \\
\hline A42' & A & $\mathrm{T}$ & A & A & A & A & $\mathrm{T}$ & A & A & A & A & $\mathrm{T}$ & $\mathrm{T}$ & A & - & - & - & - & - & - & - & - & - & - & - & - & - & - & - & A & - & - & - & - & - \\
\hline A42'a & A & $\mathrm{T}$ & A & A & A & A & $\mathrm{T}$ & A & A & A & A & $\mathrm{T}$ & $\mathrm{T}$ & A & - & - & - & - & - & - & - & - & - & - & - & - & - & - & - & A & - & - & - & - & - \\
\hline $\mathbf{A 4 3}^{\prime}$ & A & $\mathrm{T}$ & A & A & A & A & $\mathrm{T}$ & A & A & A & A & $\mathrm{T}$ & $\mathrm{T}$ & A & - & - & - & - & - & - & - & - & - & - & - & - & - & - & - & A & - & - & - & - & - \\
\hline $\mathbf{A 4 3}$ & A & $\mathrm{T}$ & A & A & A & A & $\mathrm{T}$ & A & A & A & A & $\mathrm{T}$ & $\mathrm{T}$ & A & - & - & - & - & - & - & - & - & - & - & - & - & - & - & - & A & - & - & - & - & - \\
\hline A44 & A & $\mathrm{T}$ & A & A & A & A & $\mathrm{T}$ & A & A & A & A & $\mathrm{T}$ & $\mathrm{T}$ & A & - & - & - & - & - & - & - & - & - & - & - & - & - & - & - & A & - & - & - & - & - \\
\hline $\mathbf{A 4 5}^{\prime}$ & A & $\mathrm{T}$ & A & A & A & A & $\mathrm{T}$ & A & A & A & A & $\mathrm{T}$ & $\mathrm{T}$ & A & - & - & - & - & - & - & - & - & - & - & - & - & - & - & - & A & - & - & - & - & - \\
\hline A46' & A & $\mathrm{T}$ & A & A & A & A & $\mathrm{T}$ & A & A & A & A & $\mathrm{T}$ & $\mathrm{T}$ & A & A & 1 & 1 & 1 & $\mathrm{~A}$ & A & $\mathrm{T}$ & $\mathrm{T}$ & $\mathrm{T}$ & A & $\mathrm{T}$ & $\mathrm{T}$ & A & A & A & A & - & - & - & - & - \\
\hline
\end{tabular}

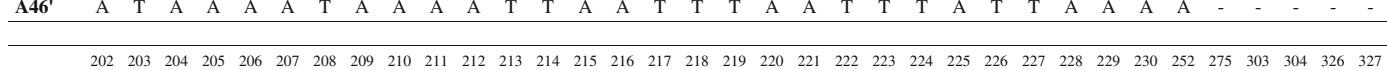

\begin{tabular}{|c|c|c|c|c|c|c|c|c|c|c|c|c|c|c|c|c|c|c|c|c|c|c|c|c|c|c|c|c|c|c|c|c|c|c|c|}
\hline & & & & & & & & & & & & & & & & & & & & & & & & & & & & & d & 5 & 6 & $\mathrm{e}$ & & 7 & $\mathrm{f}$ \\
\hline A3 & & 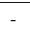 & 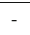 & 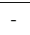 & & - & & - & - & - & - & - & - & - & - & - & - & - & - & 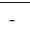 & - & - & - & - & & - & - & - & - & $\mathrm{T}$ & A & - & - & G & A \\
\hline A14 & & . & . & . & & - & - & - & - & - & - & & & - & - & - & - & - & - & & - & 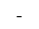 & - & - & & 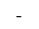 & - & - & - & $\mathrm{T}$ & C & A & $\mathrm{T}$ & G & - \\
\hline A1 & - & - & - & - & . & - & - & - & - & - & - & - & & - & - & - & - & - & - & & - & - & 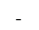 & . & & & - & - & - & $\mathrm{T}$ & $\mathrm{C}$ & & - & G & A \\
\hline A29a & - & - & - & - & - & - & - & - & - & - & - & - & - & - & - & - & - & - & - & - & - & - & - & - & - & - & - & - & - & $\mathrm{T}$ & C & - & - & $\mathrm{G}$ & A \\
\hline A30 & - & - & - & - & - & - & - & - & - & - & - & - & - & - & - & - & - & - & - & - & - & - & - & - & - & - & - & - & $\mathrm{T}$ & $\mathrm{T}$ & $\mathrm{C}$ & - & - & G & - \\
\hline A31 & - & - & - & - & - & - & - & - & - & - & - & - & - & - & - & - & - & - & - & - & - & - & - & - & - & - & - & - & $\mathrm{T}$ & $\mathrm{T}$ & C & - & - & $\mathrm{G}$ & - \\
\hline A32 & - & - & - & - & - & - & - & - & - & - & - & - & - & - & - & - & - & - & - & . & - & - & - & - & - & - & - & - & $\mathrm{T}$ & A & C & - & - & G & - \\
\hline A33 & - & - & - & - & - & - & - & - & - & - & - & - & - & - & - & - & - & - & - & - & - & - & - & - & - & - & - & - & - & $\mathrm{T}$ & C & - & - & G & - \\
\hline A34 & $\mathrm{T}$ & $\mathrm{T}$ & $\mathrm{T}$ & $\mathrm{T}$ & A & $\mathrm{T}$ & A & $\mathrm{T}$ & $\mathrm{T}$ & $\mathrm{T}$ & A & A & $\mathrm{T}$ & A & A & A & A & $\mathrm{T}$ & A & A & A & $\mathrm{T}$ & A & A & $\mathrm{T}$ & $\mathrm{T}$ & A & A & - & $\mathrm{T}$ & $\mathrm{C}$ & - & - & G & - \\
\hline A35 & - & - & - & - & - & - & - & - & - & - & - & - & - & - & - & - & - & - & - & - & - & - & - & - & - & - & - & - & - & $\mathrm{T}$ & $\mathrm{C}$ & - & - & G & A \\
\hline A36 & - & - & - & - & - & - & - & - & - & - & - & - & - & - & - & - & - & - & - & - & - & - & - & - & - & - & - & - & $\mathrm{T}$ & $\mathrm{T}$ & C & - & - & G & - \\
\hline A37 & - & - & - & - & - & - & - & - & - & - & - & - & - & - & - & - & - & - & - & - & - & - & - & - & - & - & - & - & $\mathrm{T}$ & $\mathrm{T}$ & C & - & - & G & - \\
\hline $\mathbf{A 3 8}^{\prime}$ & - & - & - & - & - & - & - & - & - & - & - & - & - & - & - & - & - & - & - & - & - & - & - & - & - & - & - & - & - & $\mathrm{T}$ & C & - & - & G & A \\
\hline 'A39' & - & - & - & - & - & - & - & - & - & - & - & - & - & - & - & - & - & - & - & - & - & - & - & - & - & - & - & - & - & $\mathrm{T}$ & C & - & - & $\mathrm{G}$ & A \\
\hline $\mathbf{A 4 0}$ & - & - & - & - & - & - & - & - & - & - & - & - & - & - & - & - & - & - & - & - & - & - & - & - & - & - & - & - & - & $\mathrm{T}$ & C & - & - & G & - \\
\hline A41 & - & - & - & - & - & - & - & - & - & - & - & - & - & - & - & - & - & - & - & 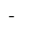 & - & - & - & - & - & - & - & - & $\mathrm{T}$ & $\mathrm{T}$ & C & - & - & A & - \\
\hline A42 & - & - & - & - & - & - & - & - & - & - & - & - & - & - & - & - & - & - & - & - & - & - & - & - & - & - & - & - & - & $\mathrm{T}$ & C & - & - & G & A \\
\hline A42'a & - & - & - & - & - & - & - & - & - & - & - & - & - & - & - & - & - & - & - & - & - & - & - & - & - & - & - & - & - & $\mathrm{T}$ & C & - & - & $\mathrm{G}$ & A \\
\hline A43' & - & - & - & - & - & - & - & - & - & - & - & - & - & - & - & - & - & - & - & - & - & - & - & - & - & - & - & - & - & $\mathrm{T}$ & C & - & - & G & A \\
\hline A43'a & - & - & - & - & - & - & - & - & - & - & - & - & - & - & - & - & - & - & - & - & - & - & - & - & - & - & - & - & - & $\mathrm{T}$ & C & - & - & G & A \\
\hline A4 & - & - & - & - & - & - & - & - & - & - & - & - & - & - & - & - & - & - & - & - & - & - & - & - & - & - & - & - & - & $\mathrm{T}$ & C & - & - & G & A \\
\hline A45 & - & - & - & - & - & - & - & - & - & - & - & - & - & - & - & - & - & - & - & - & - & - & - & - & - & - & - & - & - & $\mathrm{T}$ & $\mathrm{C}$ & - & - & G & A \\
\hline A46' & - & - & - & - & - & - & - & - & - & - & - & - & - & - & - & - & - & - & - & & - & . & - & - & & - & - & - & - & $\mathrm{T}$ & A & - & - & G & $\mathrm{A}$ \\
\hline
\end{tabular}

Figure 3. Variable sites of tRNAleu-cox 2 intergenic region of the 16 novel haplotypes, the two additional sequence variants (A42'a and A43'a), and the previously reported African haplotypes (A3, A14, A16, A29a, A30). Sequences of A29a (FJ890930.1) and A30 (EF033654.1) were obtained from GenBank. Sequences of A3, A14, and A16 were obtained from individuals collected in Portugal. The numbers on the top line represent the nucleotide position within the tRNA ${ }^{\text {leu }}$-cox2 sequenced region. Position 1 marks the beginning of the tRNA ${ }^{\text {leu }}$ gene, corresponding to position 3370 of the honey bee mitochondrial genome (Crozier and Crozier 1993). Substitution sites are numbered from 1 to 17. Indels (marked with a dash) are denoted by letters from $a$ to $q$. The 15-bp deletion characteristic of the $\mathrm{P}_{1}$ element is marked as $d 1$ (position 115). Variable sites between positions 158-358, 365-550, and 551-714 are within Q1, Q2, and Q3 elements, respectively. Position 745 identifies the beginning of Q4. Full sequences are available in GenBank under accession numbers JQ746684-JQ746701. 


\begin{tabular}{|c|c|c|c|c|c|c|c|c|c|c|c|c|c|c|c|c|c|c|c|c|c|c|c|c|c|c|c|c|c|c|c|c|c|c|}
\hline & 328 & 329 & 344 & 345 & 346 & 347 & 348 & 349 & 350 & 351 & 352 & 353 & 354 & 355 & 356 & 357 & 358 & 365 & 387 & 406 & 429 & 435 & 448 & 457 & 471 & 536 & 537 & 538 & 539 & 540 & 541 & 542 & 543 & 544 \\
\hline & & g & h & & & & & & & & & & & & 8 & & & $\mathbf{i}$ & 9 & 10 & $\mathbf{j}$ & $\mathbf{k}$ & 11 & 12 & 13 & 1 & & & & & & & & \\
\hline A3 & G & A & A & $\mathrm{T}$ & $\mathrm{T}$ & $\mathrm{T}$ & A & A & $\mathrm{T}$ & $\mathrm{T}$ & $\mathrm{T}$ & A & $\mathrm{T}$ & $\mathrm{T}$ & A & A & A & - & G & C & $\mathrm{T}$ & $\mathrm{T}$ & $\mathrm{T}$ & C & C & A & $\mathrm{T}$ & $\mathrm{T}$ & $\mathrm{T}$ & A & A & $\mathrm{T}$ & $\mathrm{T}$ & $\mathrm{T}$ \\
\hline A14 & - & - & A & $\mathrm{T}$ & $\mathrm{T}$ & $\mathrm{T}$ & A & A & $\mathrm{T}$ & $\mathrm{T}$ & $\mathrm{T}$ & A & $\mathrm{T}$ & $\mathrm{T}$ & A & A & A & $\mathrm{C}$ & A & $\mathrm{T}$ & - & $\mathrm{T}$ & $\mathrm{T}$ & C & $\mathrm{C}$ & A & $\mathrm{T}$ & $\mathrm{T}$ & $\mathrm{T}$ & A & A & $\mathrm{T}$ & $\mathrm{T}$ & T \\
\hline A16 & $\mathrm{G}$ & A & A & $\mathrm{T}$ & $\mathrm{T}$ & $\mathrm{T}$ & A & A & $\mathrm{T}$ & $\mathrm{T}$ & $\mathrm{T}$ & A & $\mathrm{T}$ & $\mathrm{T}$ & A & A & A & - & G & $\mathrm{T}$ & - & $\mathrm{T}$ & $\mathrm{T}$ & C & C & A & $\mathrm{T}$ & $\mathrm{T}$ & $\mathrm{T}$ & A & A & $\mathrm{T}$ & $\mathrm{T}$ & $\mathrm{T}$ \\
\hline A29a & G & A & A & $\mathrm{T}$ & $\mathrm{T}$ & $\mathrm{T}$ & A & A & $\mathrm{T}$ & $\mathrm{T}$ & $\mathrm{T}$ & A & $\mathrm{T}$ & $\mathrm{T}$ & A & A & A & - & G & $\mathrm{T}$ & $\mathrm{T}$ & $\mathrm{T}$ & $\mathrm{T}$ & C & C & A & $\mathrm{T}$ & $\mathrm{T}$ & $\mathrm{T}$ & A & A & $\mathrm{T}$ & $\mathrm{T}$ & $\mathrm{T}$ \\
\hline A30 & - & A & A & $\mathrm{T}$ & $\mathrm{T}$ & $\mathrm{T}$ & A & A & $\mathrm{T}$ & $\mathrm{T}$ & $\mathrm{T}$ & A & $\mathrm{T}$ & $\mathrm{T}$ & A & A & A & C & G & $\mathrm{T}$ & $\mathrm{T}$ & $\mathrm{T}$ & $\mathrm{T}$ & C & C & A & $\mathrm{T}$ & $\mathrm{T}$ & $\mathrm{T}$ & A & A & $\mathrm{T}$ & $\mathrm{T}$ & $\mathrm{T}$ \\
\hline A31 & - & A & A & $\mathrm{T}$ & $\mathrm{T}$ & $\mathrm{T}$ & A & A & $\mathrm{T}$ & $\mathrm{T}$ & $\mathrm{T}$ & A & $\mathrm{T}$ & $\mathrm{T}$ & A & A & A & $\mathrm{C}$ & G & $\mathrm{T}$ & $\mathrm{T}$ & - & $\mathrm{T}$ & C & $\mathrm{C}$ & A & $\mathrm{T}$ & $\mathrm{T}$ & $\mathrm{T}$ & A & A & $\mathrm{T}$ & $\mathrm{T}$ & $\mathrm{T}$ \\
\hline A32 & - & A & A & $\mathrm{T}$ & $\mathrm{T}$ & $\mathrm{T}$ & A & A & $\mathrm{T}$ & $\mathrm{T}$ & $\mathrm{T}$ & A & $\mathrm{T}$ & $\mathrm{T}$ & A & A & A & C & G & $\mathrm{T}$ & $\mathrm{T}$ & $\mathrm{T}$ & A & C & C & A & $\mathrm{T}$ & $\mathrm{T}$ & $\mathrm{T}$ & A & A & $\mathrm{T}$ & $\mathrm{T}$ & $\mathrm{T}$ \\
\hline A33 & - & A & A & $\mathrm{T}$ & $\mathrm{T}$ & $\mathrm{T}$ & A & A & $\mathrm{T}$ & $\mathrm{T}$ & $\mathrm{T}$ & A & $\mathrm{T}$ & $\mathrm{T}$ & A & A & A & C & G & $\mathrm{T}$ & $\mathrm{T}$ & $\mathrm{T}$ & A & C & C & A & $\mathrm{T}$ & $\mathrm{T}$ & $\mathrm{T}$ & A & A & $\mathrm{T}$ & $\mathrm{T}$ & $\mathrm{T}$ \\
\hline A34 & - & A & A & $\mathrm{T}$ & $\mathrm{T}$ & $\mathrm{T}$ & A & A & $\mathrm{T}$ & $\mathrm{T}$ & $\mathrm{T}$ & A & $\mathrm{T}$ & $\mathrm{T}$ & A & A & A & C & A & $\mathrm{T}$ & - & $\mathrm{T}$ & $\mathrm{T}$ & $\mathrm{C}$ & $\mathrm{C}$ & A & $\mathrm{T}$ & $\mathrm{T}$ & $\mathrm{T}$ & A & A & $\mathrm{T}$ & $\mathrm{T}$ & $\mathrm{T}$ \\
\hline A35 & G & A & A & $\mathrm{T}$ & $\mathrm{T}$ & $\mathrm{T}$ & A & A & $\mathrm{T}$ & $\mathrm{T}$ & $\mathrm{T}$ & A & $\mathrm{T}$ & $\mathrm{T}$ & A & A & A & - & G & $\mathrm{T}$ & $\mathrm{T}$ & $\mathrm{T}$ & A & C & C & A & $\mathrm{T}$ & $\mathrm{T}$ & $\mathrm{T}$ & A & A & $\mathrm{T}$ & $\mathrm{T}$ & $\mathrm{T}$ \\
\hline A36 & G & A & A & $\mathrm{T}$ & $\mathrm{T}$ & $\mathrm{T}$ & A & A & $\mathrm{T}$ & $\mathrm{T}$ & $\mathrm{T}$ & A & $\mathrm{T}$ & $\mathrm{T}$ & A & A & A & $\mathrm{C}$ & G & $\mathrm{T}$ & $\mathrm{T}$ & $\mathrm{T}$ & $\mathrm{T}$ & C & $\mathrm{C}$ & A & $\mathrm{T}$ & $\mathrm{T}$ & $\mathrm{T}$ & A & A & $\mathrm{T}$ & $\mathrm{T}$ & $\mathrm{T}$ \\
\hline A37 & - & A & A & $\mathrm{T}$ & $\mathrm{T}$ & $\mathrm{T}$ & A & A & $\mathrm{T}$ & $\mathrm{T}$ & $\mathrm{T}$ & A & $\mathrm{T}$ & $\mathrm{T}$ & A & A & A & C & A & $\mathrm{T}$ & $\mathrm{T}$ & $\mathrm{T}$ & $\mathrm{T}$ & C & C & A & $\mathrm{T}$ & $\mathrm{T}$ & $\mathrm{T}$ & A & A & $\mathrm{T}$ & $\mathrm{T}$ & $\mathrm{T}$ \\
\hline $\mathbf{A 3 8}^{\prime}$ & G & A & A & $\mathrm{T}$ & $\mathrm{T}$ & $\mathrm{T}$ & A & A & $\mathrm{T}$ & $\mathrm{T}$ & $\mathrm{T}$ & A & $\mathrm{T}$ & $\mathrm{T}$ & $\mathrm{T}$ & A & A & - & G & $\mathrm{T}$ & $\mathrm{T}$ & $\mathrm{T}$ & $\mathrm{T}$ & C & C & A & $\mathrm{T}$ & $\mathrm{T}$ & $\mathrm{T}$ & A & A & $\mathrm{T}$ & $\mathrm{T}$ & $\mathrm{T}$ \\
\hline $\mathbf{A 3 9}^{\prime}$ & G & A & A & $\mathrm{T}$ & $\mathrm{T}$ & $\mathrm{T}$ & A & A & $\mathrm{T}$ & $\mathrm{T}$ & $\mathrm{T}$ & A & $\mathrm{T}$ & $\mathrm{T}$ & A & A & A & - & G & $\mathrm{T}$ & $\mathrm{T}$ & $\mathrm{T}$ & $\mathrm{T}$ & C & $\mathrm{C}$ & A & $\mathrm{T}$ & $\mathrm{T}$ & $\mathrm{T}$ & A & A & $\mathrm{T}$ & $\mathrm{T}$ & $\mathrm{T}$ \\
\hline $\mathbf{A 4 0 ^ { \prime }}$ & - & A & A & $\mathrm{T}$ & $\mathrm{T}$ & $\mathrm{T}$ & A & A & $\mathrm{T}$ & $\mathrm{T}$ & $\mathrm{T}$ & A & $\mathrm{T}$ & $\mathrm{T}$ & A & A & A & C & A & $\mathrm{T}$ & - & $\mathrm{T}$ & $\mathrm{T}$ & C & C & - & - & - & - & - & - & - & - & - \\
\hline A41 & - & A & - & - & - & - & - & - & - & - & - & - & - & - & - & - & - & $\mathrm{C}$ & A & $\mathrm{T}$ & $\mathrm{T}$ & $\mathrm{T}$ & $\mathrm{T}$ & C & $\mathrm{C}$ & A & $\mathrm{T}$ & $\mathrm{T}$ & $\mathrm{T}$ & A & A & $\mathrm{T}$ & $\mathrm{T}$ & $T$ \\
\hline A42' & G & A & A & $\mathrm{T}$ & $\mathrm{T}$ & $\mathrm{T}$ & A & A & $\mathrm{T}$ & $\mathrm{T}$ & $\mathrm{T}$ & A & $\mathrm{T}$ & $\mathrm{T}$ & A & A & A & - & G & $\mathrm{T}$ & $\mathrm{T}$ & $\mathrm{T}$ & $\mathrm{T}$ & C & $\mathrm{T}$ & A & $\mathrm{T}$ & $\mathrm{T}$ & $\mathrm{T}$ & A & A & $\mathrm{T}$ & $\mathrm{T}$ & $\mathrm{T}$ \\
\hline A42'a & G & A & A & $\mathrm{T}$ & $\mathrm{T}$ & $\mathrm{T}$ & A & A & $\mathrm{T}$ & $\mathrm{T}$ & $\mathrm{T}$ & A & $\mathrm{T}$ & $\mathrm{T}$ & A & A & A & - & G & $\mathrm{T}$ & $\mathrm{T}$ & $\mathrm{T}$ & $\mathrm{T}$ & C & C & A & $\mathrm{T}$ & $\mathrm{T}$ & $\mathrm{T}$ & A & A & $\mathrm{T}$ & $\mathrm{T}$ & $\mathrm{T}$ \\
\hline $\mathbf{A 4 3}^{\prime}$ & G & A & A & $\mathrm{T}$ & $\mathrm{T}$ & $\mathrm{T}$ & A & A & $\mathrm{T}$ & $\mathrm{T}$ & $\mathrm{T}$ & A & $\mathrm{T}$ & $\mathrm{T}$ & A & A & A & - & A & $\mathrm{T}$ & $\mathrm{T}$ & - & $\mathrm{T}$ & C & C & A & $\mathrm{T}$ & $\mathrm{T}$ & $\mathrm{T}$ & A & A & $\mathrm{T}$ & $\mathrm{T}$ & $\mathrm{T}$ \\
\hline A43' $\mathbf{a}$ & G & A & A & $\mathrm{T}$ & $\mathrm{T}$ & $\mathrm{T}$ & A & A & $\mathrm{T}$ & $\mathrm{T}$ & $\mathrm{T}$ & A & $\mathrm{T}$ & $\mathrm{T}$ & A & A & A & - & A & $\mathrm{T}$ & $\mathrm{T}$ & $\mathrm{T}$ & $\mathrm{T}$ & C & C & A & $\mathrm{T}$ & $\mathrm{T}$ & $\mathrm{T}$ & A & A & $\mathrm{T}$ & $\mathrm{T}$ & $\mathrm{T}$ \\
\hline A44' & - & A & A & $\mathrm{T}$ & $\mathrm{T}$ & $\mathrm{T}$ & A & A & $\mathrm{T}$ & $\mathrm{T}$ & $\mathrm{T}$ & A & $\mathrm{T}$ & $\mathrm{T}$ & A & A & A & - & G & $\mathrm{T}$ & $\mathrm{T}$ & $\mathrm{T}$ & $\mathrm{T}$ & C & C & A & $\mathrm{T}$ & $\mathrm{T}$ & $\mathrm{T}$ & A & A & $\mathrm{T}$ & $\mathrm{T}$ & $\mathrm{T}$ \\
\hline A45" & G & A & A & $\mathrm{T}$ & $\mathrm{T}$ & $\mathrm{T}$ & A & A & $\mathrm{T}$ & $\mathrm{T}$ & $\mathrm{T}$ & A & $\mathrm{T}$ & $\mathrm{T}$ & A & A & A & - & G & $\mathrm{T}$ & - & $\mathrm{T}$ & $\mathrm{T}$ & C & C & A & $\mathrm{T}$ & $\mathrm{T}$ & $\mathrm{T}$ & A & A & $\mathrm{T}$ & $\mathrm{T}$ & $\mathrm{T}$ \\
\hline $\mathrm{A}^{\prime} 6^{\prime}$ & G & A & A & $\mathrm{T}$ & $\mathrm{T}$ & $\mathrm{T}$ & A & A & $T$ & $T$ & $T$ & A & $\mathrm{T}$ & $T$ & A & A & A & & G & C & $T$ & $T$ & $\mathrm{~T}$ & $T$ & $\mathrm{C}$ & A & $\mathrm{T}$ & $\mathrm{T}$ & $\mathrm{T}$ & A & A & $T$ & $\mathrm{~T}$ & $\mathrm{~T}$ \\
\hline
\end{tabular}

\begin{tabular}{|c|c|c|c|c|c|c|c|c|c|c|c|c|c|c|c|c|}
\hline & & 546 & 547 & 548 & 549 & 550 & 551 & 557 & 579 & 593 & 621 & 640 & 709 & 713 & 714 & \\
\hline & & & & & & & $\mathrm{m}$ & $\mathrm{n}$ & 14 & 15 & o & 16 & 17 & $\mathrm{p}$ & & \\
\hline $\mathbf{A 3}$ & & $\mathrm{T}$ & $\mathrm{T}$ & A & A & A & A & C & & & $\mathrm{T}$ & $\mathrm{T}$ & & & & \\
\hline & & & & & & & & & & & & & & & & \\
\hline & 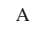 & . & & & & & & & G & C & $\mathrm{T}$ & $\mathrm{T}$ & A & & & \\
\hline & A & $\mathrm{T}$ & 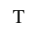 & $A$ & & & & C & G & C & $\mathrm{T}$ & $\mathrm{T}$ & & - & & \\
\hline & A & T & T & A & A & A & - & - & - & - & - & - & & & & \\
\hline & I & $\mathrm{T}$ & T & A & A & A & - & - & - & - & - & - & - & - & & \\
\hline & A & $\mathrm{T}$ & $\mathrm{T}$ & $A$ & A & F & - & - & - & - & - & - & - & - & - & \\
\hline & I & T & & $A$ & & & - & - & - & - & - & - & - & - & - & \\
\hline & & $\mathrm{T}$ & $T$ & $A$ & & A & - & - & - & - & - & - & - & - & - & \\
\hline & A & $\mathrm{T}$ & $\mathrm{T}$ & A & A & A & - & - & - & - & - & - & - & - & - & \\
\hline & . & T & & 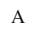 & & & . & - & - & - & - & - & - & - & & \\
\hline & A & $\mathrm{T}$ & & A & & A & - & - & - & - & - & - & - & - & & \\
\hline & A & $\mathrm{T}$ & $\mathrm{T}$ & A & A & A & A & C & G & C & $\mathrm{T}$ & $\mathrm{T}$ & A & - & - & \\
\hline & A & $\mathrm{T}$ & $\mathrm{T}$ & A & A & A & A & C & G & $\mathrm{T}$ & $\mathrm{T}$ & $\mathrm{T}$ & A & - & - & \\
\hline & & - & - & - & & - & A & C & A & C & - & $\mathrm{T}$ & A & A & G & \\
\hline & A & $\mathrm{T}$ & $\mathrm{T}$ & A & A & A & A & C & G & C & $\mathrm{T}$ & $\mathrm{T}$ & A & - & - & \\
\hline & A & $\mathrm{T}$ & $\mathrm{T}$ & A & A & A & A & C & G & C & $\mathrm{T}$ & A & A & - & - & \\
\hline & A & $\mathrm{T}$ & $\mathrm{T}$ & A & $A$ & A & A & $\mathrm{C}$ & G & C & $\mathrm{T}$ & A & A & - & - & \\
\hline & A & $\mathrm{T}$ & $\mathrm{T}$ & A & A & A & A & - & G & C & $\mathrm{T}$ & $\mathrm{T}$ & A & - & - & \\
\hline & A & $\mathrm{T}$ & $\mathrm{T}$ & A & A & A & A & - & G & C & $\mathrm{T}$ & $\mathrm{T}$ & G & - & - & \\
\hline & A & $\mathrm{T}$ & T & A & A & A & A & C & & C & $\mathrm{T}$ & $\mathrm{T}$ & A & - & & \\
\hline & A & $\mathrm{T}$ & $\mathrm{T}$ & A & A & A & A & $\mathrm{C}$ & G & $\mathrm{C}$ & $\mathrm{T}$ & $\mathrm{T}$ & A & - & - & \\
\hline $4^{\prime}$ & $A$ & T & 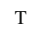 & $A$ & & & & C & & & $\mathrm{T}$ & T & A & & & \\
\hline
\end{tabular}

Figure 3. (continued).

scribed A29a is central to this group, showing considerably more connections (eight) than any other haplotype in the network. This central haplotype is connected to the closest ones by a single mutation (1-bp deletion in Q2 for A16, and a substitution in Q3 for A42'a) and to the most distant (A41') by eight mutational steps and one hypothetical node. The previously described A3 haplotype of sub-lineage $\mathrm{A}_{\mathrm{I}}$ (as defined by Franck et al. 2001) and the novel A46' are together in a branch that is separated from A29a by three and four mutations, respectively. These two sub-lineage $\mathrm{A}_{\mathrm{I}}$ haplotypes differ from each other by one transition 


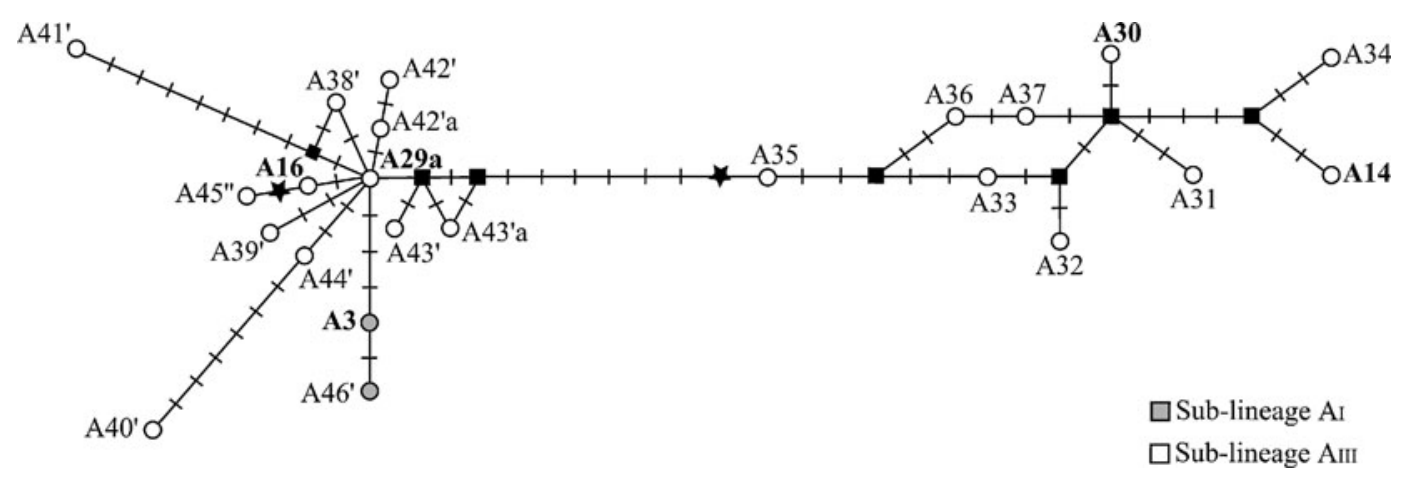

Figure 4. Median-joining network of the novel and previously described African haplotypes (marked in bold) that were identified from the tRNA ${ }^{\text {leu }}$-cox 2 region. Haplotypes are denoted as circles. Hypothetical (unsampled or extinct) haplotypes are symbolized as filled squares. The cross lines along branches represent mutational steps between nodes. The duplication of the Q element is represented by a star.

mutation $(\mathrm{C} / \mathrm{T})$, which accounted for an additional DraI recognition site in Q2 of haplotype A46' (substitution 12 in Figure 3).

\section{DISCUSSION}

This study further highlights the Atlantic side of the Iberian Peninsula as an important repository of Iberian honey bee maternal diversity. Analysis of Portuguese honey bee populations with the DraI test revealed 16 novel haplotypes of African ancestry, which were confirmed by sequence data of the tRNA ${ }^{\text {leu }}$ cox 2 intergenic region. These haplotypes join the 27 African haplotypes previously described (De la Rúa et al. 1998, 2005; Franck et al. 2001; Collet et al. 2006) of which 17 have been found in the Iberian Peninsula (Garnery et al. 1998a; De la Rúa et al. 2004, 2005; Cánovas et al. 2008), representing an increase of $59 \%$ and $94 \%$, respectively. Most haplotypes (15) contained the $\mathrm{P}_{1}$ element, typical of sublineage $\mathrm{A}_{\text {III }}$, whereas only one was assigned to sub-lineage $A_{I}$, as defined by Franck et al. (2001). The 15 haplotypes were added to the eight previously reported for sub-lineage $\mathrm{A}_{\mathrm{III}}$ (De la Rúa et al. 1998; Franck et al. 2001; Collet et al. 2006), representing an increase of $188 \%$.

The Iberian honey bee has been one of the most intensively surveyed subspecies in its natural range (Garnery et al. 1995, 1998a; Franck et al.
1998; Arias et al. 2006; Miguel et al. 2007; Cánovas et al. 2008). Therefore, detection of such a remarkable number of novel haplotypes, mostly of sub-lineage $A_{\text {III }}$ ancestry, was unexpected and suggests that prior studies have missed an important diversity component hold by the western populations of Iberia. This study not only adds to the complexity of the Iberian honey bee diversity patterns (Franck et al. 1998; Cánovas et al. 2008) but also reinforces the Atlantic distribution proposed for sub-lineage

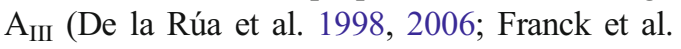
2001), as most colonies were detected in the north of continental Portugal (Figure 1), which exhibits a more Atlantic climate contrasting with the more Mediterranean southern Portugal. Our findings further support an ancient natural colonization of the Iberian Peninsula by African swarms (De la Rúa et al. 2002, 2004; Cánovas et al. 2008) as the hypothesis of historical humanmediated multiple introductions (Franck et al. 1998; Garnery et al. 1998a) is untenable with such complex levels of diversity.

Among the 16 novel haplotypes, A42 is probably the oldest because of its higher frequency and wider geographical distribution (sole haplotype shared between mainland and island colonies). Alternatively, it could have been disseminated by human-assisted colony transportation, as occurred with A29. While the history of introductions of A29 (Collet et al. 
2006; Prada et al. 2009) and its variant A29a (Szalanski and Magnus 2010) is unknown, we postulate that these haplotypes descend from colonies of Portuguese origin. The PCR-RFLP patterns of A29 (47/93/866) and A29a (47/93/ $867)$ are virtually indistinguishable from those of A16 (47/93/866). Additionally, sequence data shows that they are closely related (Figure 4 and sequences on GenBank), suggesting that A29 (Collet et al. 2006) and 29a (Szalanski and Magnus 2010) are merely sequence variants of A16. The PCR-RFLP haplotype A16 has only been reported in the Portuguese territory, including mainland (Garnery et al. 1998a; Miguel et al. 2007) and islands (De la Rúa et al. 2006). Therefore, it is possible that haplotypes A29 and A29a descend from Portuguese colonies introduced by settlers in South America in historical times and later expanded to North America (Szalanski and Magnus 2010) by Africanized honey bees. Alternatively, there were multiple independent, historical or recent, introductions in North and South America of putatively Portuguese-derived colonies.

Similarities in primary and secondary structures between the Q element and the 3' end of COI gene (5' end of Q), tRNA ${ }^{\text {leu }}$ gene (middle part of Q), and the $\mathrm{P}$ sequence ( $3^{\prime}$ end of $\mathrm{Q}$ ) led Cornuet et al. (1991) to propose an origin of the Q element by tandem replication. The first and the second $\mathrm{Q}$ of haplotypes $\mathrm{A} 41^{\prime}$ and $\mathrm{A} 40^{\prime}$, respectively, bear a $3^{\prime}$ end similar to $\mathrm{P}_{1}$ whereas those of the other Q's are similar to $\mathrm{P}_{0}$. Interestingly, the $3^{\prime}$ end of Q1 of some lineage $\mathrm{M}$ haplotypes (M34-HQ337456.1; M43Q-HQ260365.1) and Z haplotypes (Z1HM236204.1; Z12-HM236212.1; Z13HM236213.1; Z1Q-HM236205.1) also bear a deletion motif similar to the $\mathrm{P}_{1}$ element. While this deletion has probably multiple independent origins, this finding deserves further investigation as it may shed some light in the evolution of this complex region.

This study further expands on the complexity of the Iberian honey bee patterns and reinforces the importance of this southernmost European territory as a reservoir of $A$. mellifera genetic diversity. In response to a rapidly changing world (e.g., new pests and parasites, land use change), which has had severe consequences in apiculture, there is a growing alert for protecting honey bee genetic resources across its natural range (Jensen et al. 2005; De la Rúa et al. 2009; Dietemann et al. 2009; Haddad et al. 2009) and an increasing number of conservation programs, specially to protect $A$. m. mellifera (reviewed by De la Rúa et al. 2009). Preservation of honey bee genetic variation is a pre-requisite for longterm adaptive change and avoidance of fitness decline, through inbreeding depression, and thereby a guarantee of a sustainable apiculture. The Iberian Peninsula has been a stage for evolutionary events that have shaped the evolutionary history of western European honey bee lineage. Therefore, this territory certainly deserves special attention in both small- and large-scale conservation programs.

\section{ACKNOWLEDGMENTS}

We are indebted to numerous technicians of the Divisões de Intervenção Veterinária (Direcção Geral de Veterinária) and of the Beekeeping Associations, who kindly provided the honey bee samples. Financial support was provided by Fundação para a Ciência e Tecnologia and COMPETE/QREN/EU (project PTDC/BIA-BEC/ 099640/2008), Programa Apícola Nacional (Medida 6A) to M. A. Pinto, and by Fundación Séneca (project 11961/PI/09) to P. De la Rúa. We also thank the two anonymous reviewers for their valuable comments.

La façade atlantique de la péninsule ibérienne : un « point chaud " pour une nouvelle diversité maternelle de type africain chez l'abeille

Abeille ibérienne / diversité génétique / ADN mitochondrial / test DraI / Portugal

Die atlantische Seite der iberischen Halbinsel: ein Hotspot für bisher unbekannte Afrika-typische maternale Diversität bei Honigbienen

Iberische Honigbiene / genetische Diversität / mtDNA / DraI-Test / Portugal 


\section{REFERENCES}

Arias, M.C., Rinderer, T.E., Sheppard, W.S. (2006) Further characterization of honey bees from the Iberian Peninsula by allozyme, morphometric and mtDNA haplotype analyses. J. Apic. Res. 45, 188-196

Bandelt, H.J., Forster, P., Rohl, A. (1999) Medianjoining networks for inferring intraspecific phylogenies. Mol. Biol. Evol. 16, 37-48

Cánovas, F., De la Rúa, P., Serrano, J., Galián, J. (2008) Geographical patterns of mitochondrial DNA variation in Apis mellifera iberiensis (Hymenoptera: Apidae). J. Zool. Syst. Evol. Res. 46, 24-30

Cánovas, F., De la Rúa, P., Serrano, J., Galián, J. (2011) Microsatellite variability reveals beekeeping influences on Iberian honeybee populations. Apidologie. 42, 235-251

Collet, T., Ferreira, K.M., Arias, M.C., Soares, A.E.E., Del Lama, M.A. (2006) Genetic structure of Africanized honeybee populations (Apis mellifera L.) from Brazil and Uruguay viewed through mitochondrial DNA COI-COII patterns. Heredity. 97, 329-335

Cornuet, J.M., Fresnaye, J. (1989) Biometrical study of honey bee populations from Spain and Portugal. Apidologie. 20, 93-101

Cornuet, J.M., Garnery, L., Solignac, M. (1991) Putative origin and function of the intergenic region between $\mathrm{COI}$ and COII of Apis mellifera L. mitochondrial DNA. Genetics. 128, 393-403

Crozier, R.H., Crozier, Y.C. (1993) The mitochondrial genome of the honey bee Apis mellifera: complete sequence and genome organization. Genetics. 133, 97-117

De la Rúa, P., Serrano, J., Galián, J. (1998) Mitochondrial DNA variability in the Canary islands honeybees (Apis mellifera L.). Mol. Ecol. 7, 1543-1547

De la Rúa, P., Galián, J., Serrano, J., Moritz, R.F.A. (2001) Molecular characterization and population structure of the honeybees from the Balearic islands (Spain). Apidologie. 32, 417-427

De la Rúa, P., Galián, J., Serrano, J., Moritz, R.F.A. (2002) Microsatellite analysis of non-migratory colonies of Apis mellifera iberica from south-eastern Spain. J. Zool. Syst. Evol. Research. 40, 164-168

De la Rúa, P., Galián, J., Serrano, J., Moritz, R.F.A. (2003) Genetic structure of Balearic honeybee populations based on microsatellite polymorphism. Genet. Sel. Evol. 35, 339-350

De la Rúa, P., Jiménez, Y., Galián, J., Serrano, J. (2004) Evaluation of the biodiversity of honey bee (Apis mellifera) populations from eastern Spain. J. Apic. Res. 43, 162-166

De la Rúa, P., Hernandez-Garcia, R., Jiménez, Y., Galián, J., Serrano, J. (2005) Biodiversity of Apis mellifera iberica (Hymenoptera: Apidae) from northeastern Spain assessed by mitochondrial analysis. Insect Syst. Evol. 36, 21-28

De la Rúa, P., Galián, J., Pedersen, B.V., Serrano, J. (2006) Molecular characterization and population structure of Apis mellifera from Madeira and the Azores. Apidologie. 37, 699-708

De la Rúa, P., Jaffé, R., Dall'Olio, R., Muñoz, I., Serrano, J. (2009) Biodiversity, conservation and current threats to European honeybees. Apidologie. 40, 263-284

Dietemann, V., Pirk, C.W.W., Crewe, R. (2009) Is there a need for conservation of honeybees in Africa? Apidologie. 40, 285-295

Engel, M.S. (1999) The taxonomy of recent and fossil honey bees (Hymenoptera: Apidae; Apis). J. Hym. Res. 8, 165-196

Franck, P., Garnery, L., Solignac, M., Cornuet, J.M. (1998) The origin of west European subspecies of honeybees (Apis mellifera): new insights from microsatellite and mitochondrial data. Evolution. 52, 1119-1134

Franck, P., Garnery, L., Loiseau, A., Oldroyd, B.P., Hepburn, H.R., Solignac, M., Cornuet, J.M. (2001) Genetic diversity of the honey bee in Africa: microsatellite and mitochondrial data. Heredity. 86, 420-430

Garnery, L., Solignac, M., Celebrano, G., Cornuet, J.M. (1993) A simple test using restricted PCRamplified mitochondrial DNA to study the genetic structure of Apis mellifera L. Experientia. 49, $1016-1021$

Garnery, L., Mosshine, E.H., Oldroyd, B.P., Cornuet, J.M. (1995) Mitochondrial DNA variation in Moroccan and Spanish honey bee populations. Mol. Ecol. 4, 465-471

Garnery, L., Franck, P., Baudry, E., Vautrin, D., Cornuet, J.M., Solignac, M. (1998a) Genetic diversity of the west European honey bee (Apis mellifera mellifera and $A$. m. iberica). I. Mitochondrial DNA. Genet. Sel. Evol. 30, S31-S47

Garnery, L., Franck, P., Baudry, E., Vautrin, D., Cornuet, J.M., Solignac, M. (1998b) Genetic diversity of the west European honey bee (Apis mellifera mellifera and A. m. iberica). II. Microsatellite loci. Genet. Sel. Evol. 30, S49-S74

Gómez, A., Lunt, D.H. (2007). Refugia within refugia: patterns of phylogeographic concordance in the Iberian Peninsula. Springer, Dordrecht

Haddad, N., Meixner, M.D., Fuchs, S., Migdadi, H., Garnery, L., Sheppard, W.S. (2009) Mitochondrial DNA support for genetic reserves of Apis mellifera syriaca in Jordan. J. Apic. Res. 48, 19-22

Hewitt, G.M. (1999) Post-glacial re-colonization of European biota. Biol. J. Linn. Soc. 68, 87-112

Hewitt, G.M. (2001) Speciation, hybrid zones and phylogeography — or seeing genes in space and time. Mol. Ecol. 10, 537-549 
Jensen, A.B., Palmer, K.A., Boomsma, J.J., Pedersen, B.V. (2005) Varying degrees of Apis mellifera ligustica introgression in protected populations of the black honeybee, Apis mellifera mellifera, in northwest Europe. Mol. Ecol. 14, 93-106

Miguel, I., Iriondo, M., Garnery, L., Sheppard, W.S., Estonba, A. (2007) Gene flow within the M evolutionary lineage of Apis mellifera: role of the Pyrenees, isolation by distance and post-glacial recolonization routes in the western Europe. Apidologie. 38, 141-155

Miguel, I., Baylac, M., Iriondo, M., Manzano, C., Garnery, L., Estonba, A. (2010) Both geometric morphometric and microsatellite data consistently support the differentiation of the Apis mellifera M evolutionary branch. Apidologie. 42, 150-161

Prada, Q.C.F., Duran, J.T., Salamanca, G.G., Del Lama, M.A. (2009) Population genetics of Apis mellifera L. (Hymenoptera: Apidae) from Columbia. J. Apic. Res. 48, 3-10

Rortais, A., Arnold, G., Alburaki, M., Legout, H., Garnery, L. (2011) Review of the DraI COI-COII test for the conservation of the black honeybee (Apis mellifera mellifera). Conserv. Genet. Resour. 3, 383-391
Ruttner, F., Tassencourt, L., Louveaux, J. (1978) Biometrical-statistical analysis of the geographic variability of Apis mellifera L. Apidologie. 9, 363-381

Sheppard, W.S., Meixner, M.D. (2003) Apis mellifera pomonella, a new honey bee subspecies from Central Asia. Apidologie. 34, 367-375

Smith, D.R., Glenn, T.C. (1995) Allozyme polymorphisms in spanish honeybees (Apis mellifera iberica). J. Hered. 86, 12-16

Smith, D.R., Palopoli, M.F., Taylor, B.R., Garnery, L., Cornuet, J.M., Solignac, M., Brown, W.M. (1991) Geographical overlap of two mitochondrial genomes in Spanish honeybees (Apis mellifera iberica). J. Hered. 82, 96-100

Szalanski, A.L., Magnus, R.M. (2010) Mitochondrial DNA characterization of Africanized honey bee (Apis mellifera L.) populations from the USA. J. Apic. Res. 49, 177-185

Tamura, K., Peterson, D., Peterson, N., Stecher, G., Nei, M., Kumar, S. (2011) MEGA5: Molecular Evolutionary Genetics Analysis using Maximum Likelihood, Evolutionary Distance, and Maximum Parsimony Methods. Mol. Biol. Evol. doi:10.1093/molbev/msr121 